\title{
Stellar coronagraphy with prolate apodized circular apertures
}

\author{
R. Soummer ${ }^{1}$, C. Aime $^{1}$, and P. E. Falloon ${ }^{2}$ \\ ${ }^{1}$ UMR 6525 Astrophysique, Faculté des Sciences, Université de Nice Sophia Antipolis, Parc Valrose, 06108 Nice Cedex 2, \\ France \\ e-mail: Remi .Soummer@unice.fr;Claude.Aime@unice.fr \\ 2 School of Physics, The University of Western Australia, Crawley, WA 6009, Australia \\ e-mail: falloon@physics.uwa.edu.au
}

Received 16 September 2002 / Accepted 25 October 2002

\begin{abstract}
This paper generalizes to circular apertures the theoretical study of stellar coronagraphy with prolate apodized rectangular entrance apertures of Aime et al. (2002). The main difference between the two studies is that circular prolate spheroidal functions are used for a circular aperture instead of linear prolate spheroidal functions for rectangular apertures. Owing to the radial property of the problem, the solution to the general equation for coronagraphy is solved using a Hankel transform instead of a product of Fourier transforms in the rectangular case. This new theoretical study permits a better understanding of coronagraphy, stressing the importance of entrance pupil apodization. A comparison with the classical unapodized Lyot technique is performed: a typical gain of $10^{4}$ to $10^{6}$ can be obtained theoretically with this technique. Circular and rectangular apertures give overall comparable results: a total extinction of the star light is obtained for Roddier \& Roddier's phase mask technique whilst optimal starlight rejections are obtained with a Lyot opaque mask. A precise comparison between a circular aperture and a square aperture of same surface favors the use of a circular aperture for detection of extrasolar planets.
\end{abstract}

Key words. instrumentation: high angular resolution - techniques: high angular resolution - stars: planetary systems

\section{Introduction}

The interest in stellar coronagraphy is relatively recent (Bonneau et al. 1975; Smith \& Terrile 1984; Malbet 1996; Beuzit et al. 1997; Mouillet et al. 1997), and nowadays mainly stimulated by the exoplanets searches. The direct imaging of an exoplanet is the most exciting objective for high contrast imaging techniques: it will provide valuable information such as its mass (removing the ambiguity due to the radial velocities technique), its albedo and its atmosphere chemical composition if spectral information can be obtained.

Several other scientific objectives, with different instrumental requirements, may also benefit from the techniques developed for high contrast imaging. Among them, the circumstellar disks for a better understanding of their dynamic and the physics of the grains (Mouillet 1997), the environment of evolved stars which could also include old planets, the Active Galactic Nucleis for a better imaging of the central jets. Since the first indirect exoplanet detection (Mayor \& Queloz 1995), several techniques have been proposed to reach high contrast imaging (Gay \& Rabbia 1996; Roddier \& Roddier 1997; Rouan et al. 2000; Abe et al. 2001).

In a previous paper (Aime et al. 2002), we analyzed the theoretical aspects of the association of coronagraphy (Lyot et al.) with entrance aperture apodization and derived the

Send offprint requests to: $\mathrm{R}$. Soummer,

e-mail: Remi.Soummer@unice.fr integral equation for total coronagraphic extinction of the star light. This integral equation depends on the shape of the apodized aperture.

For the rectangular aperture case we analyzed (Aime et al. 2001), the apodization solution is given by the linear prolate spheroidal functions discovered by Slepian \& Pollak (1961). These functions have already found many applications in optics, as well described by Frieden (1971). For coronagraphy, the relevant particularity is that these functions are invariant to a finite Fourier transform.

We showed that a theoretical total extinction can be obtained with a perfect Roddier \& Roddier coronagraph. For Lyot coronagraphy, we showed that though a total extinction is not possible, very interesting solutions can be obtained with the prolate apodized coronagraph: much smaller masks than for the classical technique are required, and very high dynamic may be obtained, depending on the mask size and apodization. Although less powerful than the phase mask technique, the Prolate Apodized Lyot Coronagraph (PALC) was found to remain interesting because it is easier to implement and much less sensitive to chromatic effects in a wide band experiment. The technique for a square aperture was also compared to the Apodized Square Aperture concept that considers apodization alone (Nisenson \& Papaliolios 2001; Soummer et al. 2002b).

The present paper generalizes the study to the case of coronagraphy with an apodized circular aperture. In this case, 
the solution for the apodization function is given by the circular prolate spheroidal functions developed by Slepian (1964) and Heurtley (1964). The complexity of the formalism was the occasion for us to go much deeper into the theoretical aspects of the problem, to find new analytical simplifications and bring a lot of interesting new results.

The present study is restricted to a perfect circular aperture, operating in space, for a monochromatic unresolved onaxis star. However, the equations considering a polychromatic source are also given and discussed. This is an aspect in which the PALC may perform better than the phase mask technique. The purpose of this study is to settle the theoretical background for circular apertures and give the ideal performance attainable for such a coronagraph. A more technical study of a realistic instrument will be realized in a future work and compared to the perfect case.

\section{Equations for coronagraphy with circular apodized apertures}

The general formalism to describe a coronagraphic experiment with an apodized aperture has been given by Aime et al. (2002). They showed that a complete extinction of the starlight could be obtained with a coronagraph implemented at the focus of an apodized telescope. Denoting $P(x, y)$ the aperture transmission function $(P(x, y)=1$ inside the aperture, and 0 outside $)$, and $\Phi(x, y)$ an apodization function $(0 \leq \Phi(x, y) \leq 1)$, the total extinction is obtained if the following equation is satisfied:

$P(x, y) \Phi(x, y) * \frac{1}{\lambda^{2} f^{2}} \widehat{M}\left(\frac{x}{\lambda f}, \frac{y}{\lambda f}\right)=\frac{1}{\varepsilon} \Phi(x, y)$,

where $\lambda$ is the wavelength, $f$ the focal length of the telescope and $M(x, y)$ a function that describes the mask shape, equal to 1 inside the coronagraphic mask and 0 outside.

With a rectangular aperture, the problem is simplified because this two dimensional equation separates into the product of two symmetric one dimensional equations. The apodization function solution is equal to 1 at the center of the rectangular aperture and decreases towards the edges as the product of two linear prolate functions aligned along the length and the width of the rectangle.

With a circular aperture, the problem is much more complicated since we cannot separate anymore the solution into two linear conditions in $x$ and $y$. Fortunately, the radial properties of the aperture and mask shape make the solution possible: a two dimensional radial function $\Phi(\boldsymbol{r})$ can be represented by its radial cut $\Phi(r)$, where $r=\sqrt{x^{2}+y^{2}}$ is the modulus of the position vector $\boldsymbol{r}=(x, y)$. For the sake of simplicity, we will use the same notation for these two functions, the interpretation being straightforward for the alert reader, depending on the context. In particular, the computations developed using Fourier transforms in the rectangular separable case, are here solved by means of Hankel transforms.

We consider a circular aperture of diameter $D$ apodized by a radial apodization function $\Phi(r)$ : $0 \leq \Phi(r) \leq 1$. We consider the four successive planes denoted A (telescope pupil), B (telescope focus, coronagraphic mask), C (relay pupil plane, Lyot Stop) and D (final focus plane)
The complex amplitude at the entrance apodized pupil plane is proportional to:

$\Psi_{\mathrm{A}}(\boldsymbol{r})=\Pi\left(\frac{r}{D}\right) \Phi(\boldsymbol{r})$

where $\Pi(r)=1$ for $|r| \leq 1 / 2$ and 0 otherwise.

The wave propagation between each planes (A, B, C, D) writes as a scaled Fourier Transform (FT) and we assume that the optical layout is properly designed to eliminate the quadratic phase terms associated with the propagation of the waves (Goodman 1996). The FT of a radial function is also a radial function, which radial cut (for $r \geq 0$ ) can be expressed using the Hankel Transform (HT):

$\widehat{\Psi}_{\mathrm{A}}(r)=\int_{0}^{\infty} 2 \pi \rho \Psi_{\mathrm{A}}(\rho) J_{0}(2 \pi \rho r) \mathrm{d} \rho$.

In plane $\mathrm{B}$, the coronagraphic mask is a disk of diameter $d$ proportional to the size of the diffraction pattern of the circular apodized aperture. At the wavelength $\lambda$, the mask size is:

$d=a \lambda f$,

where $a$ is a parameter that expresses the diameter of the mask in units of $1 / D$ and $f$ is the telescope focal length. The wave amplitude after the mask writes:

$\Psi_{\mathrm{B}}(\boldsymbol{r})=\frac{1}{\imath \lambda f} \widehat{\Psi}_{\mathrm{A}}\left(\frac{\boldsymbol{r}}{\lambda f}\right) \times\left[1-\epsilon \Pi\left(\frac{r}{d}\right)\right]$,

where the symbol denotes the HT. We do not consider in this paper other mask functions for which the mask transmission itself is also apodized (Watson et al. 1991). The parameter $\epsilon$ has only two relevant values (Aime et al. 2001, 2002): $\epsilon=1$ for an opaque mask (Lyot's coronagraphy) and $\epsilon=2$ for a $\pi$ Phase Mask (PM) (Roddier and Roddier's coronagraphy).

An optical system is then used to obtain an image of the telescope aperture in plane C. Here also, the complex amplitude of the wave is written using a scaled HT of Eq. (5). For simplicity, we assume that the focal lengths of the successive optical systems are identical (if not, an appropriate change of variables leads to a similar result). In the relay pupil plane $C$, we denote by $\Psi_{\mathrm{C}}^{-}(\boldsymbol{r})$ the amplitude before the application of the diaphragm (Lyot Stop):

$\Psi_{\mathrm{C}}^{-}(\boldsymbol{r})=\Psi_{\mathrm{A}}(\boldsymbol{r})-\epsilon \Psi_{\mathrm{A}}(r) * \frac{a J_{1}(\pi a r)}{2 r}$.

Note that the convolution product $*$ is a two-dimensional convolution product over the variables $x$ and $y$. However, this convolution of two radial functions can be simplified using the properties of the Hankel Transform, as detailed in Appendix A, to obtain for the radial cut of the wave amplitude:

$\Psi_{\mathrm{C}}^{-}(r)=\Pi\left(\frac{r}{D}\right) \Phi(r)-\epsilon(2 \pi)^{2} \int_{0}^{\frac{D}{2}} \xi \Phi(\xi) K_{0}(\xi, r) \mathrm{d} \xi$.

A diaphragm (called Lyot Stop) exactly equal to the entrance pupil shape is then set in plane $\mathrm{C}$, to remove the light diffracted outside the aperture:

$\Psi_{\mathrm{C}}^{+}(r)=\left[\Phi(r)-\epsilon(2 \pi)^{2} \int_{0}^{\frac{D}{2}} \xi \Phi(\xi) K_{0}(\xi, r) \mathrm{d} \xi\right] \times \Pi\left(\frac{r}{D}\right)$. 
The kernel $K_{0}(\xi, r)$ expression (given in Eq. (A.8) and Eq. (A.9)) permits a numerical study of circular coronagraphy, for a given aperture transmission, using a unique onedimensional integration that can be computed easily. This method is more interesting and precise than a fully numerical approach (avoiding the sampling problem of both the pupil and the mask that imposes the use of large images), with competitive computing time.

Note that the kernel $K_{0}(\xi, r)$ is analog to the sine cardinal kernel for the rectangular aperture coronagraphy (Aime et al. 2002).

\section{Circular prolate spheroidal functions as apodization functions solution to the coronagraphic problem}

Considering for example Eq. (6), the coronagraphic effect appears as a subtraction of two wave amplitudes in the relay pupil plane. Though the coronagraphic effect is expected in the final focal plane $\mathrm{D}$, it is here more convenient to consider the relay pupil plane. Ideally, we seek for an exact wave subtraction, within the entire exit pupil, or at least, the best subtraction possible.

The integral in Eq. (7) or Eq. (8) must be proportional to $\Phi(r)$, to permit an exact subtraction of the two wavefronts inside the aperture in the relay pupil plane: the apodization function $\Phi(r)$ must be the eigenfunction of this kernel.

The solutions $\Phi(r)$ are given by the circular prolate functions, which are the eigenfunctions of this eigenvalue problem. We give a brief presentation of them in Appendix B.

For the apodization function $\Phi(r)$ (normalized circular prolate function):

$\Phi(r)=\frac{\Theta(r)}{\Theta(0)}$,

associated with the eigenvalue $\Lambda$ (positive number between 0 an 1), the integral in Eq. (8) can be expressed using Eq. (B.3):

$(2 \pi)^{2} \int_{0}^{\frac{D}{2}} \xi \Phi(\xi) K_{0}(\xi, r) \mathrm{d} \xi=\Lambda \Phi(r)$

Equations (7) and (8) then find a much simpler expression:

$\Psi_{\mathrm{C}}^{-}(r)=\Phi(r) \Pi\left(\frac{r}{D}\right)-\epsilon \Lambda \Phi(r)$,

$\Psi_{\mathrm{C}}^{+}(r)=\Pi\left(\frac{r}{D}\right) \Phi(r)(1-\epsilon \Lambda)$.

Depending on the eigenvalues and on $\epsilon$, we discuss the solutions to the problem in the following subsections for Roddier \& Roddier and Lyot. A lot of valuable information can be derived analytically (throughput, impulse response, residual star intensity etc.) from the prolate properties.

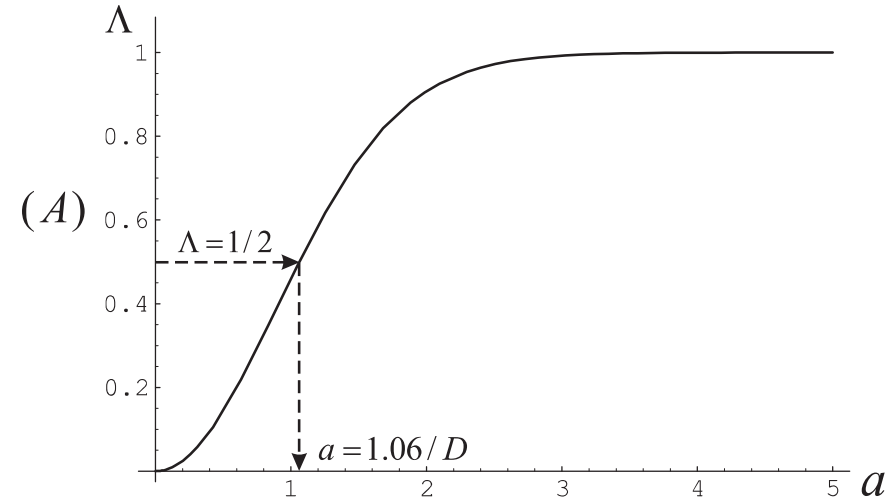

$(B)$
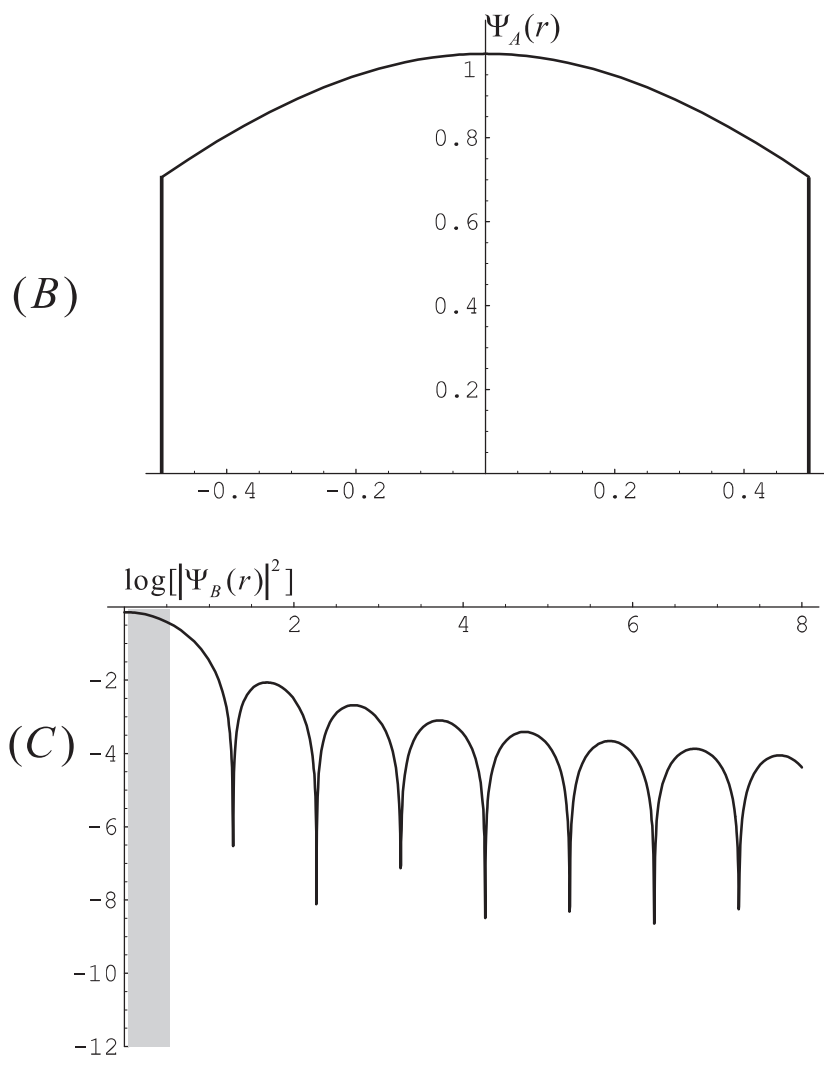

Fig. 1. Roddier \& Roddier coronagraphy with prolate entrance pupil apodization. A) Eigenvalue $\Lambda$ as a function of the mask size $a$ with the solution for $R \& R \quad \Lambda=1 / 2$. B) Corresponding circular prolate apodization, in amplitude. C) Illustration of the PSF for a planet, assuming the coronagraphic effect negligible, for a point source, sufficiently well off-axis. The grey tint rectangle shows the size of the mask, at the same scale.

\subsection{Circular prolate solution for Roddier \& Roddier coronagraphy $(\epsilon=2)$}

For Roddier \& Roddier's coronagraphy, the $\pi$ phase mask imposes $\epsilon=2$. The amplitude inside the exit pupil (Eq. (12)) becomes:

$\Psi_{\mathrm{C}}^{+}(r)=\Pi\left(\frac{r}{D}\right) \Phi(r)(1-2 \Lambda)$.

An exact solution exists (total extinction) for the eigenvalue $\Lambda=1 / 2$ and a unique mask size and apodization (eigenfunction) corresponds to this eigenvalue. From the inverse 
function of the eigenvalue curve, we obtain the mask diameter $a=1.06 / D$, as illustrated in Fig. 1 .

The transmission of the corresponding apodization is represented in amplitude in Fig. 1. This solution is in excellent agreement with the solution obtained numerically by Guyon \& Roddier (2000). A comparison between the analytical and numerical solution is made in Soummer et al. (2002a). It is also necessary to know the energy throughput of the entrance pupil. Using the orthogonality relation for circular prolate functions (Eq. (B.5)), the throughput (normalized to the throughput of the raw circular aperture) finds a simple expression:

$T=\frac{4}{\pi D^{2}} \int_{0}^{\frac{D}{2}}\left(\frac{\Theta(r)}{\Theta(0)}\right)^{2} 2 \pi r \mathrm{~d} r=\frac{4}{\pi D^{2}} \frac{2 \pi \Lambda}{\Theta^{2}(0)}$.

At first sight, this expression seem to depend on the telescope diameter. However, considering Eq. (C.1) and knowing that $\varphi(0)=0$ (Slepian 1964), we can show that $\Theta(0) \propto \frac{1}{D}$ and the relative intensity transmission is independent of the telescope diameter.

For the solution of Roddier \& Roddier, we find $T_{R R}=72.6 \%$

In the focal plane B (the phase mask plane), the amplitude of the wave (before the phase mask) can be expressed analytically, using the invariance of the circular prolate functions to a finite HT (Eq. (B.1)):

$\Psi_{\mathrm{B}}^{-}(r)=\frac{1}{\imath \lambda f} \int_{0}^{\frac{D}{2}} \Phi(\xi) J_{0}\left(2 \pi \frac{r}{\lambda f} \xi\right) 2 \pi \xi \mathrm{d} \xi$.

We obtain the following wave amplitude before the phase mask:

$\Psi_{\mathrm{B}}^{-}(r)=\frac{1}{l \lambda f} \frac{D}{a} \Lambda^{\frac{1}{2}} \Phi\left(\frac{r D}{a \lambda f}\right)$.

Reminding the notation of Eq. (4), we can stress that the wave amplitude is simply homothetic from a plane to another (invariance of the circular prolate to a finite HT):

$\Psi_{\mathrm{B}}^{-}(r)=\frac{D}{d} \Lambda^{\frac{1}{2}} \Phi\left(\frac{r D}{d}\right)$.

Moreover, the amplitude inside the phase mask $(0 \leq r \leq d / 2)$ in plane B is exactly homothetic to the amplitude inside the entrance pupil in plane A (this point was discussed in details for the rectangular aperture, Aime et al. 2002).

This expression can also be used as a good approximation for the planet response. Indeed, for an off-axis point source, situated at a distance well outside the coronagraphic mask, the extinction can be neglected and the planet focal intensity is similar to the apodized aperture without coronagraph:

$I_{\mathrm{p}}(r)=\left(\frac{D}{d}\right)^{2} \Lambda \Phi^{2}\left((r) \frac{D}{d}\right)$

This intensity for the planet (apodization alone), normalized to the impulse response maximum of the unapodized aperture is represented in logarithmic scale in Fig. 1:

$\tilde{I}_{\mathrm{p}}(r)=\left(\frac{4}{\pi D^{2}}\right)^{2} I_{\mathrm{p}}(r)$.
The grey tint rectangle represent the size of the phase mask.

Note that the loss of resolution due to the apodization of the entrance pupil is very low ( $\sim 5 \%)$ : the first zero of the impulse response is at $1.28 \lambda / D$, instead of $1.22 \lambda / D$ for the classical unapodized Airy's pattern (the apodization required for Roddier $\&$ Roddier is very light), and the equivalent widths are almost the same to $1 \%$ precision.

For this total extinction solution (Roddier \& Roddier), the wave amplitude in plane A is the truncated central part of the prolate solution $\Phi(r)$ (Eq. (2)). Considering Eq. (11), it appears that in plane $\mathrm{C}$, the wave before the Lyot stop is the complementary external part of the same prolate $\Phi(r)(\epsilon \Lambda=1$ for $R \& R)$. The conservation of energy imposes to the star energy (collected in plane A), to be totally rejected outside the pupil aperture in plane $\mathrm{C}$ (and eliminated by the Lyot stop).

This physical requirement can be checked mathematically, using the circular prolates properties, following Frieden (1971). Using Eqs. (B.7) and (B.8), with $\Lambda=1 / 2$, we can write:

$\int_{0}^{\frac{D}{2}} r|\Phi(r)|^{2} \mathrm{~d} r=\frac{1}{2} \int_{0}^{\infty} r|\Phi(r)|^{2} \mathrm{~d} r$

and straightforwardly:

$\int_{0}^{\frac{D}{2}} r|\Phi(r)|^{2} \mathrm{~d} r=\int_{\frac{D}{2}}^{\infty} r|\Phi(r)|^{2} \mathrm{~d} r$,

which is the expression of energy conservation for circular prolate functions: the energy in the central part and complementary external part of the prolate wave are equal (if $\Lambda=1 / 2$, i.e. $R \& R$ coronagraphy).

\subsection{Residual amplitude for Lyot coronagraphy $(\epsilon=1)$}

For Lyot coronagraphy, the opaque mask imposes $\epsilon=1$. The amplitude in the relay pupil plane (Eq. (12)) becomes:

$\Psi_{\mathrm{C}}^{+}(r)=\Pi\left(\frac{r}{D}\right) \Phi(r)(1-\Lambda)$,

and there is no relevant exact solution: the trivial solution $\Lambda=1$ would correspond to an infinite size opaque mask (Fig. B.2).

However, approximate solutions can be obtained for eigenvalues $\Lambda$ close to 1 and finite mask size: taking advantage of the rapid saturation of the eigenvalue curve (Fig. B.2), we can choose a mask size $a$ corresponding to an eigenvalue close to 1 , so that the residual amplitude (Eq. (22)) remains close to zero. A corresponding prolate apodization exists but is no longer an exact solution and a residual amplitude exists.

The surprising result is that the residual amplitude in the pupil plane (Eq. (22)) is itself proportional to the initial prolate function, to the factor $(1-\Lambda)$. The overall effect of Lyot coronagraphy with prolate apodization is here simply an attenuation coefficient for the on-axis point source.

The residual star intensity is then itself apodized. Moreover, in plane $\mathrm{D}$, the energy is maximally concentrated within a surface equivalent to the coronagraphic mask (prolate fundamental property Eqs. (B.7) and (B.8) (Slepian 1964; Frieden 1971). This is an important difference with classical Lyot coronagraphy, for which the residual pupil intensity in plane $\mathrm{C}$ is known 

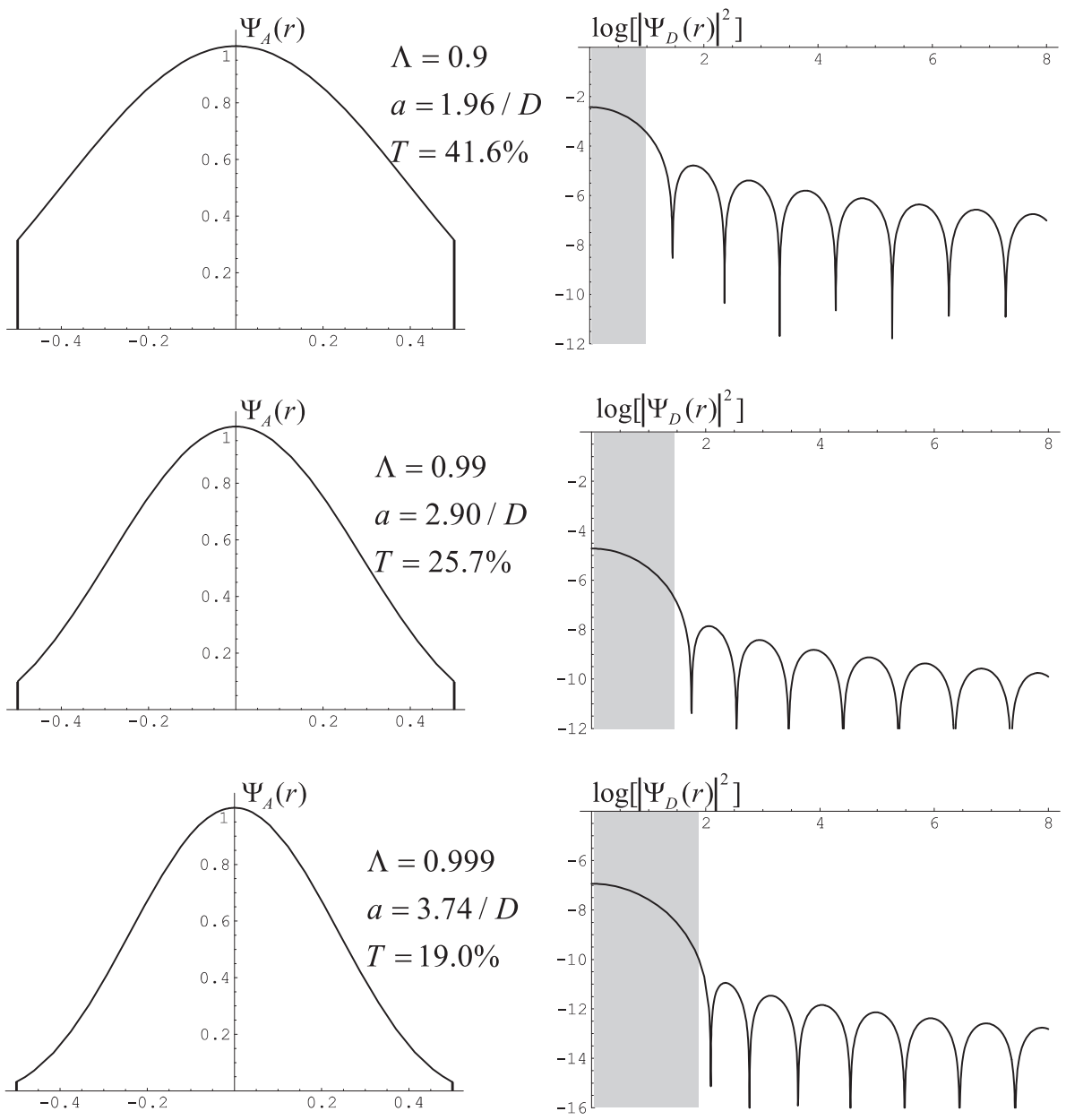

Fig. 2. Approximated solutions for Lyot coronagraphy with prolate apodization. The solutions are given for three arbitrary eigenvalues close to $1: \Lambda=0.9, \Lambda=0.99, \Lambda=$ 0.999 . For each solution, we give the radial apodization in amplitude (left), and the residual star intensity in the final focal plane (in log scale). The grey tint rectangles correspond to the mask size at the same scale. Note that the mask is always smaller than the central core.

to be maximum at the edge of the pupil (opposite effect to apodization). This is why prolate apodizations are the optimal solution for Lyot coronagraphy, in terms of maximum residual star energy concentration.

Following Eq. (22), the residual intensity for the on-axis star, is then simply reduced by the factor $(1-\Lambda)^{2}$ compared to the intensity with the apodizer alone (without the mask) Eq. (17):

$\left|\Psi_{\mathrm{D}}(r)\right|^{2}=(1-\Lambda)^{2}\left(\frac{D}{d}\right)^{2} \Lambda \Phi^{2}\left(\frac{r D}{d}\right)$.

Examples of the apodized pupil and normalized star residual intensity are given in Fig. 2, for the arbitrary eigenvalues $\Lambda=$ $0.9, \Lambda=0.99, \Lambda=0.999$ : the mask sizes are represented as grey tint rectangles and the intensity curves are normalized to the maximum of the intensity for the unapodized pupil:

$\left|\tilde{\Psi}_{\mathrm{D}}(r)\right|^{2}=\left(\frac{4}{\pi D^{2}}\right)^{2} \times\left|\Psi_{\mathrm{D}}(r)\right|^{2}$

An important point to notice is that with entrance pupil prolate apodization, the Lyot mask size is always smaller than the core of the diffraction pattern. This can be seen in Fig. 2, and also in Fig. 3 (bottom). This is a very important difference with classical Lyot coronagraphy for which the mask extends over several Airy rings.
The integrated residual energy is simply reduced by the factor $(1-\Lambda)^{2}$ compared to the throughput $T$ of the apodizer alone (Eq. (14)):

$E=\frac{4}{\pi D^{2}} \frac{2 \pi \Lambda(1-\Lambda)^{2}}{\Theta^{2}(0)}=(1-\Lambda)^{2} \times T$.

The closest to 1 the eigenvalue lie, the higher the coronagraphic star energy rejection (Fig. 3 top). However, a tradeoff exists: the transmission of the entrance pupil (Fig. 3 center) and the angular resolution (Fig. 3 bottom) decrease with $\Lambda$. The three examples of Fig. 2 are indicated in these figures with the dotted lines.

The integrated star residual energy is not the most pertinent criterion to evaluate the performance, since the high contrast imaging problem comes from the central source diffracted light. A better criterion is to consider the fractional energy in the residual star diffraction wings, relatively to the instrument throughput:

$e_{\text {wings }}^{(1)}=\frac{\int_{d / 2}^{\infty}\left|\Psi_{\mathrm{D}}(r)\right|^{2} 2 \pi r \mathrm{~d} r}{\int_{0}^{\infty}\left|\Psi_{\mathrm{A}}(r)\right|^{2} 2 \pi r \mathrm{~d} r}$.

Combining with Eq. (25), we obtain:

$e_{\text {wings }}^{(1)}=(1-\Lambda)^{2} \frac{\int_{d / 2}^{\infty}\left|\Psi_{\mathrm{D}}(r)\right|^{2} 2 \pi r \mathrm{~d} r}{\int_{0}^{\infty}\left|\Psi_{\mathrm{D}}(r)\right|^{2} 2 \pi r \mathrm{~d} r}$. 
Normalized residual energy of the star : $(1-\Lambda)^{2}$
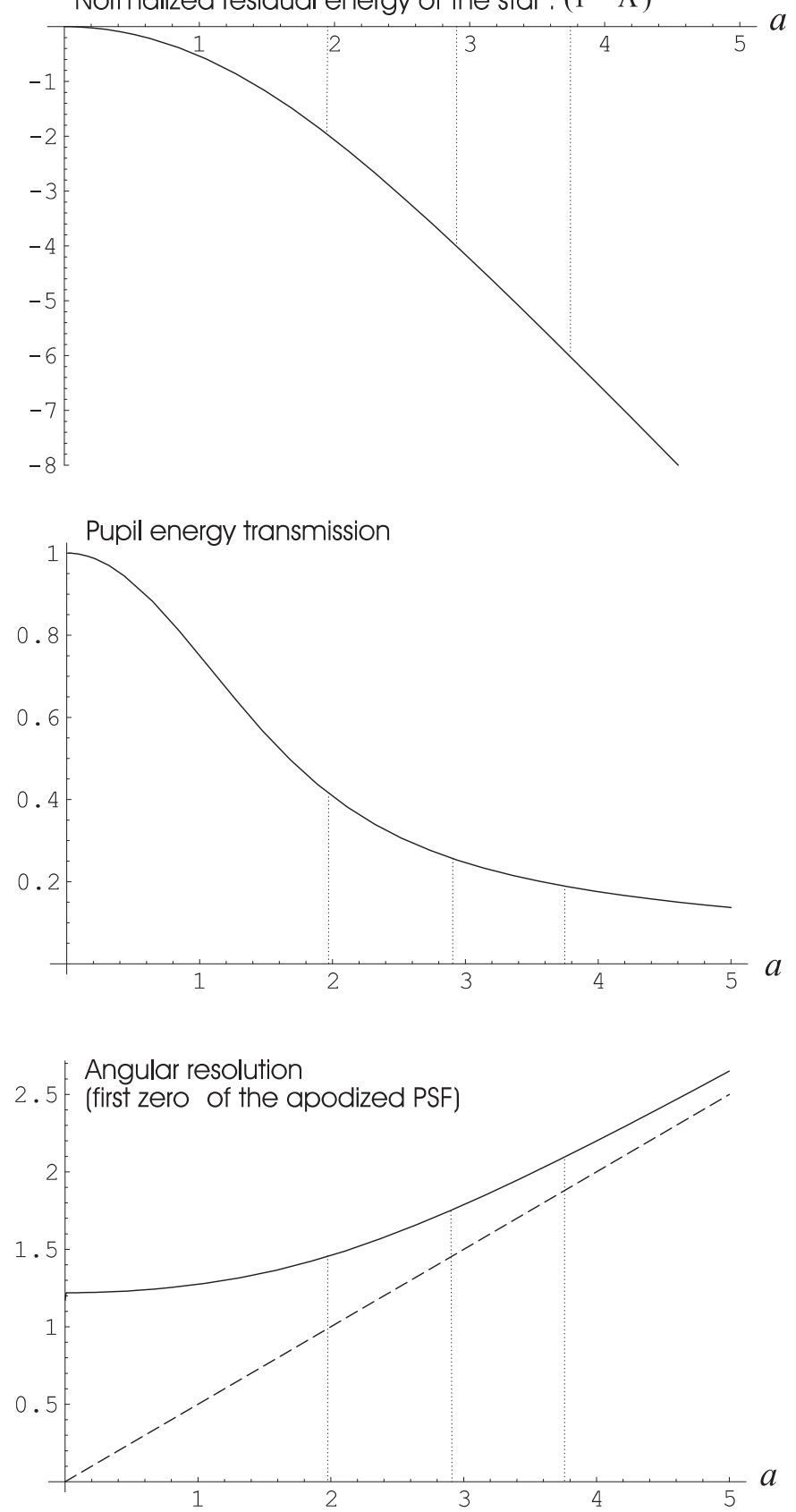

Fig. 3. Lyot coronagraphy with increasing mask size (and increasing eigenvalues and apodization strength). Top: residual energy for the star, normalized to the entrance pupil transmission. Center: illustration of the loss of transmission for the approximate solutions due to the increasing strength of the apodization. Bottom: illustration of the loss of resolution due to the apodization. The full line represents the position of the first zero of the PSF and can be compared to the half width of the mask (Dashed line). The three values at $a=1.96,2.90$ and 3.74 correspond to the examples shown in Fig. 2.

Using the properties of encircled energy for the prolate functions (Eqs. (B.7) and (B.8)), we obtain simply:

$e_{\text {wings }}^{(1)}=(1-\Lambda)^{3}$.

This criterion can be used to compare the difference of the performance between the classical Lyot coronagraph and the prolate apodized Lyot coronagraph. For the classical unapodized technique, this fractional energy is computed by a numerical integration:

$e_{\text {wings }}^{(2)}=\frac{4}{\pi \zeta^{2} D^{2}}\left(\int_{0}^{\zeta \frac{D}{2}} 2 \pi r \Psi_{\mathrm{C}}^{(\mathrm{L})}(r)^{2} \mathrm{~d} r-\int_{0}^{\frac{d}{2}} 2 \pi r \Psi_{\mathrm{D}}^{(\mathrm{L})}(r)^{2} \mathrm{~d} r\right)$,

where $\zeta$ is a coefficient for the Lyot Stop diameter reduction. $\Psi_{\mathrm{C}}^{(\mathrm{L})}(r)$ and $\Psi_{\mathrm{D}}^{(\mathrm{L})}(r)$ are the wave amplitudes for the unapodized Lyot coronagraph. We remind that for classical unapodized Lyot coronagraph, the residual intensity in the exit pupil is concentrated at the edges of the pupil and a reduction of the diameter (Lyot Stop) permits a better rejection. A comparison of $e_{\text {wings }}^{(1)}\left(\right.$ Eq. (28)) and $e_{\text {wings }}^{(2)}$ (Eq. (29)) is made in Fig. 4 for same mask sizes. The comparison is made for the Lyot stop diameters of $100 \%, 90 \%$ and $80 \%$. For example, with a mask size of $3 \lambda / D$, the efficiency of the prolate technique is roughly $10^{4}$ better than for the classical Lyot technique.

The parameters and performances for these different techniques are summarized in Table 1, including typical values for the residual diffracted light at different positions $(3 \lambda / D, 5 \lambda / D$ and $7 \lambda / D)$.

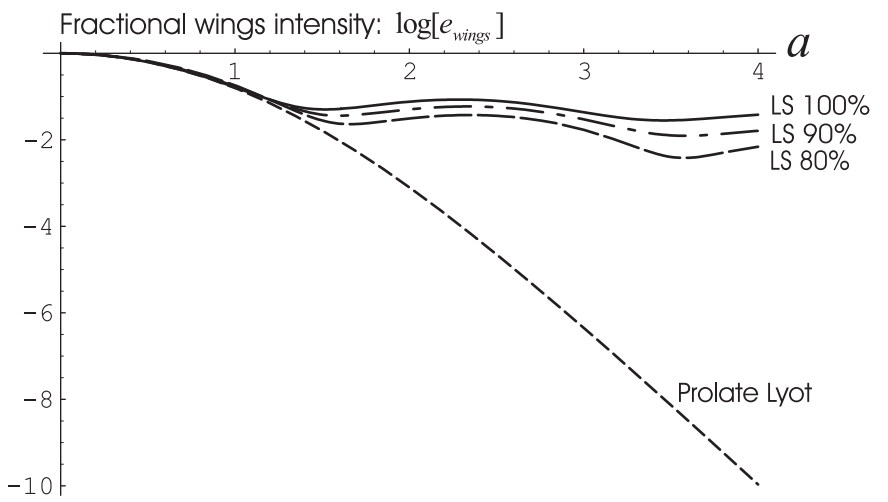

Fig. 4. Comparison of the fractional wings energy (in log scale) for classical unapodized Lyot and prolate apodized Lyot, as a function of the mask size. Full line (top curve): unapodized Lyot without Lyot stop reduction. The two similar curves underneath correspond to a Lyot Stop reduction of $90 \%$ and $80 \%$ respectively. The dashed line (bottom curve) corresponds to the prolate apodized coronagraph.

\section{Comparison between square and cicular prolate apodized aperture with Lyot coronagraph}

In this section, we compare the technique of coronagraphy with prolate apodization for square or circular apertures of same collecting surface $S$, i.e. a square aperture of length $L=D \sqrt{\pi} / 2$.

With Roddier \& Roddier coronagraphy, both shapes can perform a total extinction: in the case of a square aperture, the apodization solution consists of a product of linear prolate functions whilst for the circular aperture, the apodization is a circular prolate function. An illustration of the solutions for Roddier \& Roddier is given in Fig. 5: the apodization (in amplitude) and the impulse response without coronagraph (or the response for a planet well outside the mask). The corresponding 
Table 1. Comparison of the performances for the technique with prolate apodization and the classical unapodized Lyot technique with Lyot Stop (LS) reduction, expressed in percents. In this table, we give the values of the parameters introduced in the text.

\begin{tabular}{lllllllll}
\hline \hline Technique & eigenvalue & Throughput & Mask & Residual & Wings & level @ & level @ & level @ \\
& $\Lambda$ & $T$ & $a$ & starlight & $e_{\text {wings }}$ & $3 \lambda / D$ & $5 \lambda / D$ & $7 \lambda / D$ \\
\hline R\&R Prolate & $1 / 2$ & $72.6 \%$ & $1.06 / \mathrm{D}$ & 0 & 0 & 0 & 0 & 0 \\
Lyot Prolate & 0.9 & $41.6 \%$ & $1.96 / \mathrm{D}$ & $10^{-2}$ & $10^{-3}$ & $3 \times 10^{-6}$ & $10^{-6}$ & $10^{-7}$ \\
Lyot Prolate & 0.99 & $25.7 \%$ & $2.90 / \mathrm{D}$ & $10^{-4}$ & $10^{-6}$ & $3 \times 10^{-9}$ & $3 \times 10^{-10}$ & $10^{-10}$ \\
Lyot Prolate & 0.999 & $19.0 \%$ & $3.74 / \mathrm{D}$ & $10^{-6}$ & $10^{-9}$ & $3 \times 10^{-12}$ & $5 \times 10^{-13}$ & $10^{-13}$ \\
Lyot 100\% LS & - & $100 \%$ & $4 / \mathrm{D}$ & $4.8 \times 10^{-2}$ & $3.8 \times 10^{-2}$ & $1.6 \times 10^{-4}$ & $3.2 \times 10^{-4}$ & $5 \times 10^{-5}$ \\
Lyot 90\% LS & - & $81 \%$ & $4 / \mathrm{D}$ & $1.7 \times 10^{-2}$ & $1.6 \times 10^{-2}$ & $1.6 \times 10^{-5}$ & $6.3 \times 10^{-5}$ & $4.0 \times 10^{-6}$ \\
Lyot 80\% LS & - & $64 \%$ & $4 / \mathrm{D}$ & $9.0 \times 10^{-3}$ & $6.9 \times 10^{-3}$ & $1.6 \times 10^{-5}$ & $5.0 \times 10^{-5}$ & $2.5 \times 10^{-6}$ \\
\hline
\end{tabular}
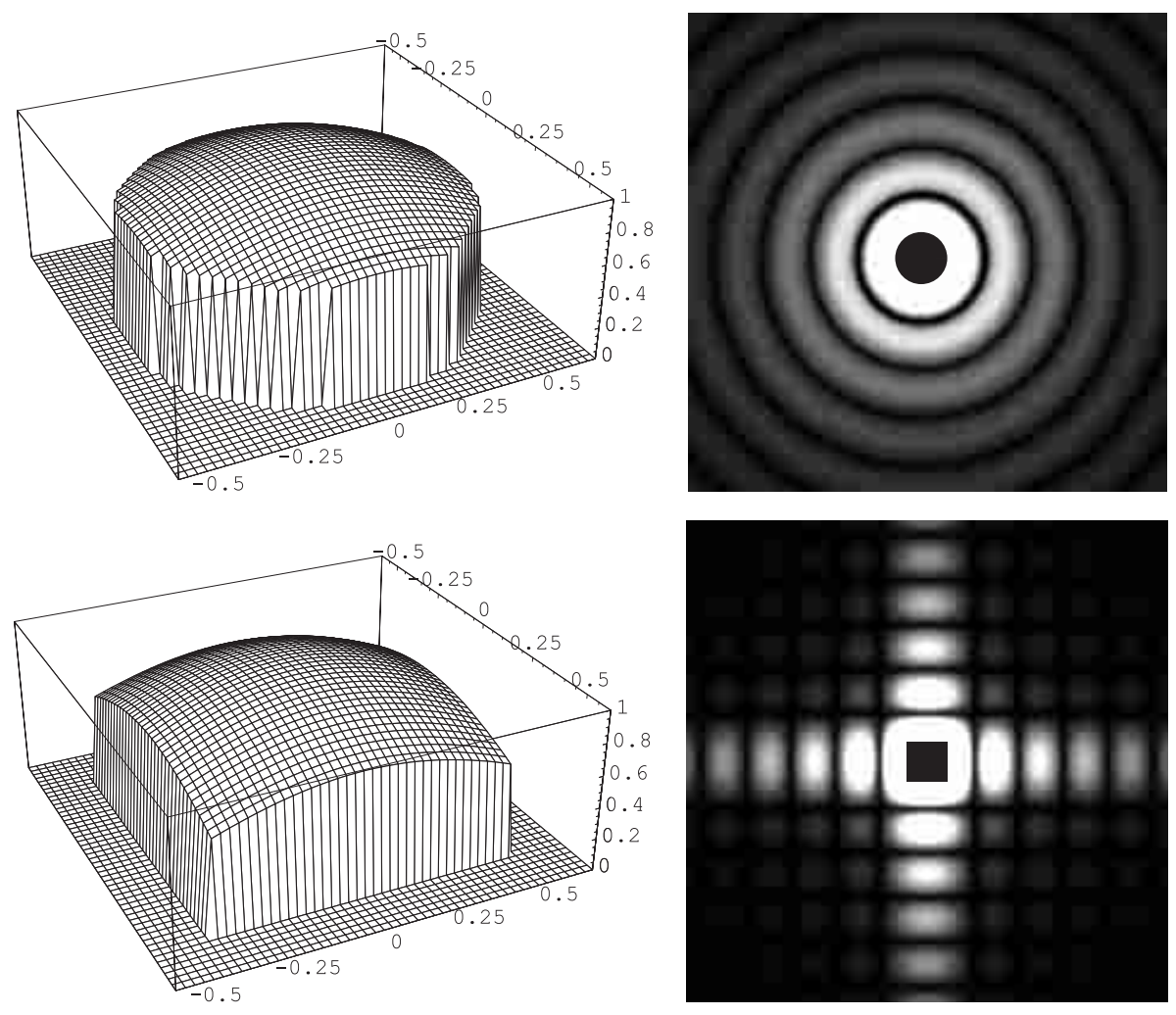

Fig. 5. Amplitude aperture transmission (left) and PSF (right), represented in Plane B, for Roddier \& Roddier coronagraphy, and a circular (top) or square (bottom) aperture. The $\pi$ phase mask is shown as a black square and disk. Both aperture can perform a total nulling of the star light. Mask sizes are: $1.06 / D$ for the disk diameter and $0.85 / L=0.96 / D$ for the length of the square. The circular aperture presents a slightly better telescope throughput (73\% instead of $70 \%$ ). The intensity scale for the PSF is not linear, but identical for the 2 figures. phase masks are also shown in the figures as a black disk and square. The surface of the circular mask $(0.69 / S)$ is slightly smaller than that of the square mask $(0.72 / S)$. The throughputs are similar, with again a slight advantage for the circular solutions ( $73 \%$ vs. $70 \%)$.

The comparison is also interesting for Lyot coronagraphy. Let us first remind the expression of the residual star intensity for a square aperture and a Lyot coronagraph, already given by Aime et al. (2002). We assume a symmetrical configuration for the square aperture of length $L$ (square coronagraphic mask of size $m$ with the same eigenvalues $\Lambda_{x}=\Lambda_{y}=\Lambda_{\mathrm{s}}$ ):

$\left|\Psi_{\mathrm{D}}(x, y)\right|^{2}=\left(1-\Lambda_{\mathrm{s}}^{2}\right)^{2}\left(\frac{\Lambda_{\mathrm{s}}}{m}\right)^{2} L^{2} \Phi^{2}\left(\frac{L}{m} x\right) \Phi^{2}\left(\frac{L}{m} y\right)$.

The residual star intensity is then itself a product of linear prolate functions and the spatial intensity is not radially uniform in the final focal plane. Compared to the circular aperture, the square aperture produces a lower residual diffracted level along a diagonal cut but a higher level along an axial cut.
A comparison of residual intensities of the two configurations is made in Figs. 6 and 7, for an arbitrary identical throughput of $30 \%$. In that case, the mask sizes are the following: the diameter of the disk is $2.6 / D$ and the length of the square is $2 / L$ (or 2.26/D for the square aperture of same surface. The surface of the disk is slightly larger than that of the square (about $4 \%$ ). The shapes of the residual intensities are quite different.

This can be explained easily as follows: comparing Eqs. (23) and (30), we see that the coronagraphic effect, for an on-axis point source, is simply the multiplication of the focal PSF without coronagraph by the factors $\left(1-\Lambda_{\mathrm{s}}^{2}\right)^{2}$ and $\left(1-\Lambda_{c}\right)^{2}$, respectively for a square and circular aperture. These values are different for a same throughput. This is clearly visible in Fig. 6 where the residual level intensity at the center of the pattern are $1.4 \times 10^{-3}$ for the square aperture and only $5.9 \times 10^{-4}$ for the circular one. Then the behavior of the residual light is given by the telescope PSF: the square aperture presents a lower residual intensity in four quadrants, but two strong diffraction patterns along its main axes. 

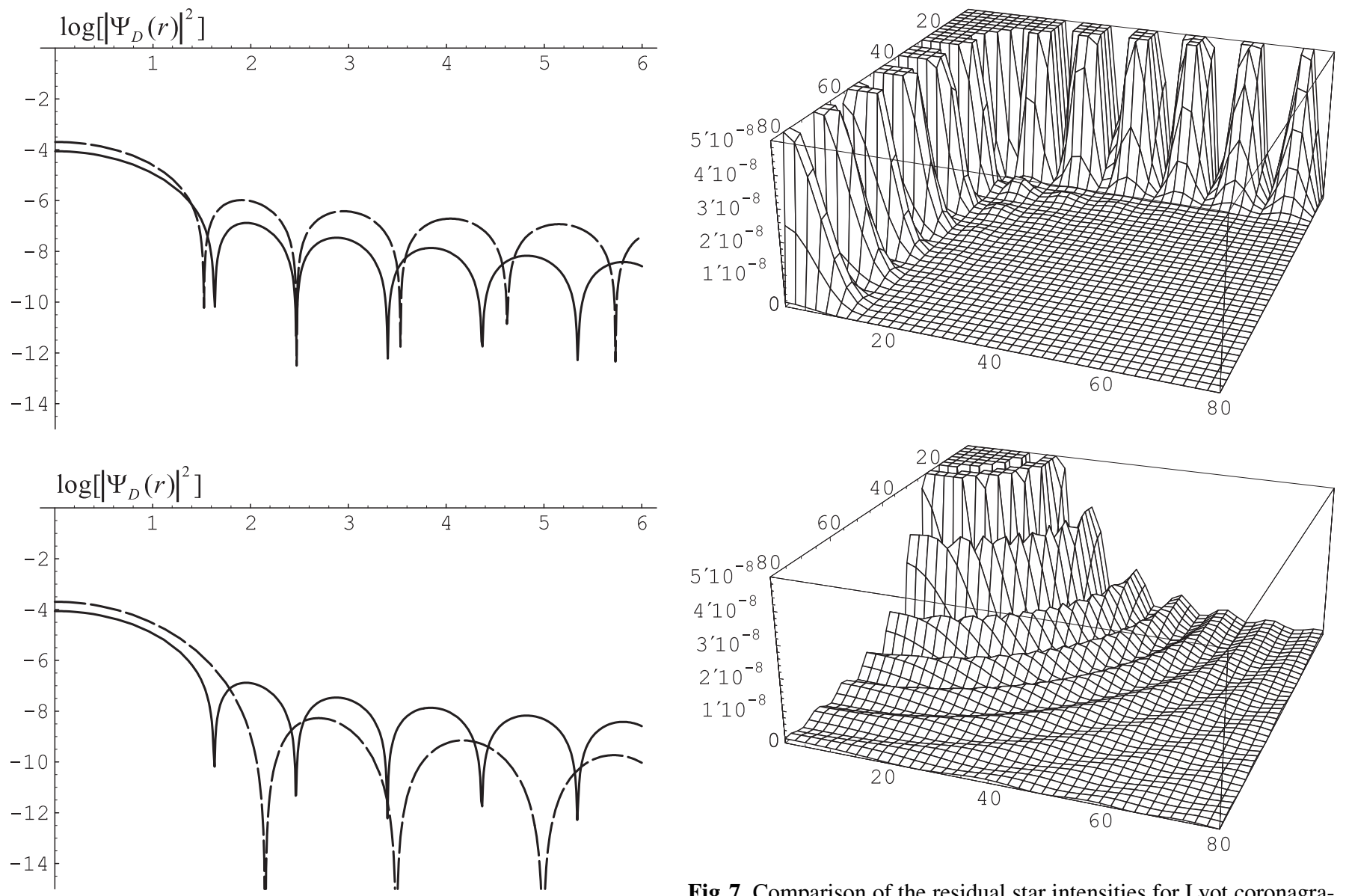

Fig. 6. Comparison of the residual star intensities (in log scale) for Lyot coronagraphy with prolate apodizations for square apertures (dashed lines) and circular apertures (full lines identical in the two figures). Curves are drawn for apertures of same surface and an identical throughput of $30 \%$. Top: circular aperture and square aperture (axial cut). Bottom: circular aperture and square aperture (diagonal cut).

The fractional wings energy, introduced above (Eq. (26)) can also be used as a criterion for a comparison between the residual diffracted levels for the circular and square apertures. The expressions for the fractional wings energy are respectively:

$e_{\text {wings }}^{(1)}=\left(1-\Lambda_{\mathrm{c}}\right)^{3}$;

$e_{\text {wings }}^{(3)}=\left(1-\Lambda_{\mathrm{s}}^{2}\right)^{3}$.

Note that the eigenvalues for the square $\left(\Lambda_{\mathrm{s}}\right)$ and circular $\left(\Lambda_{\mathrm{c}}\right)$ aperture are different for a same intensity throughput $\mathrm{T}$. For example, for $T=50 \%, \Lambda_{\mathrm{s}}=0.89$ and $\Lambda_{\mathrm{c}}=0.82$. For a given transmission $\mathrm{T}$, we compare the two fractional wings energy of Eq. (31) in Fig. 8.

\section{Discussion}

In this paper we have developed a theoretical study for coronagraphy of circular apertures with entrance pupil apodization, following the previous study for rectangular apertures (Aime et al. 2002). All the results for the rectangular configuration

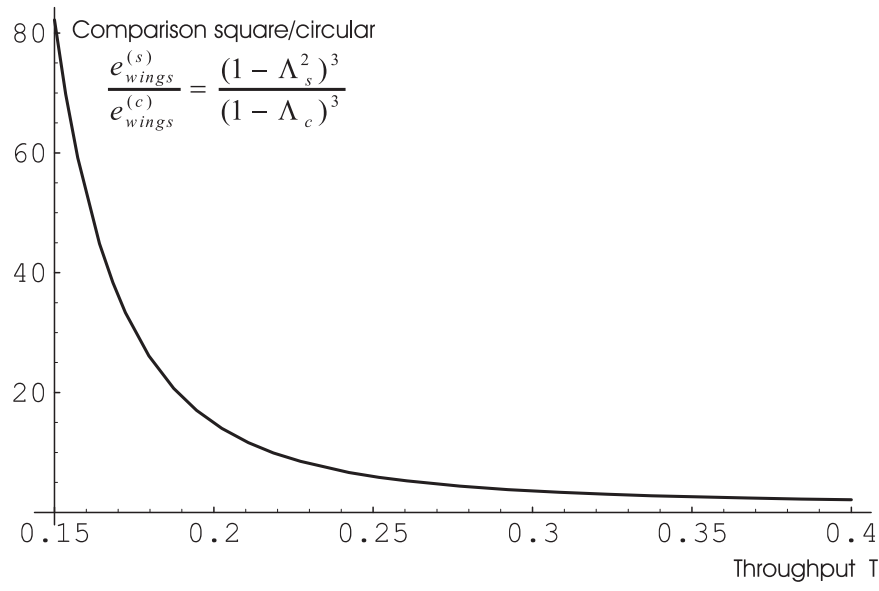

Fig. 8. Comparison for Lyot coronagraphy with prolate apodizations between square apertures and circular apertures (same surface) as a function of the throughput of the entrance apodization. The curve represents the ratio between the fractional wings energy for the square and the circular apertures. The circular aperture gives a lower residual energy in the diffraction wings, especially with increasing eigenvalues (respectively decreasing throughput).

Fig. 7. Comparison of the residual star intensities for Lyot coronagraphy with prolate apodizations for square apertures and circular apertures. The two apertures have the same surface, and the same intensity The $30 \%$ of the apodizer. The square aperture has a lowe residual intensity along a diagonal cut, but a higher residual intensity along an axial cut, than the circular aperture which is radially uniform.

(respectively decreasing throughput). 
find their analog with circular apertures. The formalism is more complex, but the physical contents are similar. We have shown that the natural apodization functions for Lyot's coronagraphy and Roddier \& Roddier's coronagraphy are the circular prolate spheroidal functions. We have proven that a total extinction of a monochromatic unresolved on-axis star can be obtained theoretically using a circular aperture apodized by circular prolate functions and Roddier \& Roddier's phase mask coronagraphy.

The study we have made is for a monochromatic wave front. R\&R's coronagraphy suffers from a double chromatism problem, the size chromatism and the phase shift chromatism compared to other phase shifting techniques, such as the wavelength-independent CIA (Gay \& Rabbia 1996; Baudoz et al. 2000a; Baudoz et al. 2000b) or the less sensitive 4QC technique (Rouan et al. 2000), or the achromatic PKC (Abe et al. 2001, 2003). However, these techniques have other drawbacks: the CIA does not produces images, and the 4QC/PKC has a lower efficiency along the axis.

For Lyot coronagraphy, only a size chromatism exits: the mask size is adapted to the wavelength $\lambda_{0}$. At the wavelength $\lambda$, the diffraction pattern is not of the same size and the mask size is not correct. Equation (6) can be re-written, with the mask size:

$a=a_{0} \frac{\lambda_{0}}{\lambda}$,

where $a_{0}$ is the mask size at the wavelength $\lambda_{0}$. The kernel equation Eq. (8) may then be used for the computation. If the bandpass is not to large, the chromatic effect for Lyot is light. Numerical simulations of these chromatic effects can be found in Soummer et al. (2002a). However, to overcome this problem for large bandpass, one can use an achromatization system that produces a magnification of the focal image proportional to $1 / \lambda$ over the bandpass (Wynne 1979). Then the aperture image must undergo the same procedure (in the reverse sense) for the Lyot stop, using a second Wynne corrector with opposite effect. Wynne correctors have been used experimentally to extend the bandwidth of speckle interferometry (Wynne 1979; Roddier et al. 1980), or for the Dark Speckles coronagraphy experiment (Boccaletti et al. 1998). A known limitation of Wynne correctors is their very small field of view. This may be acceptable for high contrast imaging applications. A detailed study of the optical design including the two Wynne correctors remains necessary to assess the performances of the whole experiment, over a given bandwidth.

For the achromatized Lyot technique, only a partial extinction of the star can be obtained, but there also, prolate apodizations give optimal results. Both the star and the planet appear in the final image with the same Point Spread Function (PSF) that is optimal in terms of concentration of light. The overall effect of Lyot's coronagraphy with prolate apodization is simply to reduce the magnitude difference between the planet and its parent star. Note that the multiple stage Lyot coronagraph proposed for rectangular aperture, is then also possible for circular aperture.

The reduction factor obtained with the prolate apodization is good enough to consider Lyot's technique for high contrast imaging, mainly because of its simplicity and its lower dependence on the deviation from the perfect case: chromatism, star diameter, telescope jitter.

The comparisons we made between circular and square aperture with the prolate coronagraphy technique are in favor of the circular aperture. For a perfect prolate apodized coronagraph using the Roddier \& Roddier phase mask technique, the circular aperture provides a slightly better instrumental throughput for a smaller mask size. For Lyot coronagraphy (PALC) the results appear even more favorable to the circular aperture that gives a lower integrated residual energy in the diffraction wings, especially when a strong apodization is used. For a given throughput, the square aperture may however permit a better planet detection in four quadrants outside its main axial diffraction patterns. Because of its cross-like dead zone, delicate and time consuming 45 degrees telescope rotation will be needed to investigate the whole field. A circular aperture with a slightly lower throughput will probably be able to detect directly the presence of a planet in a shorter time. Moreover, the comparison we have done assume telescopes of same surface. For practical reason, it is probable that a square telescope would be obtained by diaphragming a circular one, which diameter equals to the diagonal of the square. In this case the circular aperture would be much more efficient. This comparison between a square and circular aperture does not apply directly to rectangular apertures, detailed in a previous study (Aime et al. 2002), since rectangular apertures present some specific interesting advantages (high angular resolution in one direction, possible $X, \lambda$ implementation for example)

This paper intended to present a theoretical and analytical approach for coronagraphy without atmosphere, using apodized apertures. Several issues have not been tackled in this paper and remain to be studied numerically. In particular, numerical simulations will be needed to evaluate the sensitivity of these formal solutions to the physical parameters: wavelength dependence, wavefront errors, mask positioning errors, angular diameter of the star, etc. It will be also interesting to check if the technique can give valuable results from the ground using an apodized aperture with adaptive optics. All these points will be studied in a more technical future work, and some of them are already presented in Soummer et al. (2002a). A comparison with the other coronagraphic techniques is also necessary; a major point will be to include signal to noise ratio as the main comparison criterion.

Acknowledgements. The authors would like to thank André Ferrari for helpful discussions.

\section{Appendix A: Formalism for the coronagraphy of circular apertures}

In Eq. (6), the two dimensional convolution product between the 2 radial functions $f(\boldsymbol{r})=\Psi_{\mathrm{A}}(\boldsymbol{r})$ and $g(\boldsymbol{r})=\frac{a J_{1}(\pi a \boldsymbol{r})}{2 \boldsymbol{r}}$ can be simplified, using the properties of FT and HT. The Fourier Transform (FT) of the convolution product is a simple product between the FTs of each functions:

$\mathcal{F}(f(\boldsymbol{r}) * g(\boldsymbol{r}))=\widehat{f}(\boldsymbol{\rho}) \times \widehat{g}(\boldsymbol{\rho})$, 
where $\rho$ denotes the radial vector in the Fourier space and $\rho$ its modulus. Since these functions are radial functions, a radial cut (denoted $\widehat{f}(\rho)$ ) and $\widehat{f}(\rho)$ can be computed by a Hankel Transform (HT). Using Eq. (2), we have, for $\rho \geq 0$ :

$\widehat{f}(\rho)=\int_{0}^{\infty} \Pi\left(\frac{r}{D}\right) \Phi(r) J_{0}(2 \pi \rho r) 2 \pi r \mathrm{~d} r$

$\widehat{g}(\rho)=\Pi\left(\frac{\rho}{a}\right)$.

The radial cut of the FT of the convolution product is then:

$$
[\mathcal{F}(f(\boldsymbol{r}) * g(\boldsymbol{r}))]_{\text {r.c. }}=\Pi\left(\frac{\rho}{a}\right) \int_{0}^{\frac{D}{2}} \Phi(r) J_{0}(2 \pi \rho r) 2 \pi r \mathrm{~d} r .
$$

Taking again the HT of this radial expression (reminding that $(H T[H T]=I d)$, we come back to the expression of the radial cut of the convolution product:

$$
\begin{array}{r}
{[f(\boldsymbol{r}) * g(\boldsymbol{r})]_{\text {r.c. }}=\int_{\rho=0}^{\frac{a}{2}} \int_{\xi=0}^{\frac{D}{2}} \Phi(\xi)} \\
\quad J_{0}(2 \pi \rho \xi) 2 \pi \xi \mathrm{d} \xi \\
\quad \times J_{0}(2 \pi \rho r) 2 \pi r \mathrm{~d} r
\end{array}
$$

that can be written, with the kernel notation:

$[f(\boldsymbol{r}) * g(\boldsymbol{r})]_{\text {r.c. }}=(2 \pi)^{2} \int_{0}^{\frac{D}{2}} \xi \Phi(\xi) K_{0}(\xi, r) \mathrm{d} \xi$

where the Kernel:

$$
K_{0}(\xi, r)=\int_{0}^{\frac{a}{2}} \rho J_{0}(2 \pi \rho \xi) J_{0}(2 \pi \rho r) \mathrm{d} \rho,
$$

was already given by Frieden (1971) in his presentation of circular prolate functions properties (see Appendix B)

An analytical expression of the Kernel can be obtained with the help of Mathematica software (Wolfram 1999):

$$
K_{0}(\xi, r)=\frac{a r J_{0}(a \pi \xi) J_{1}(a \pi r)-a \xi J_{0}(a \pi r) J_{1}(a \pi \xi)}{4 \pi\left(r^{2}-\xi^{2}\right)},
$$

which limit for $r=\xi$ equals:

$$
K_{0}(\xi, \xi)=\frac{1}{8} a^{2}\left(J_{0}(a \pi \xi)^{2}+J_{1}(a \pi \xi)^{2}\right)
$$

for $\xi=r$.

Theses analytical expressions of the Kernel make it possible to use the integral of Eq. (A.6) for the direct computation of the convolution product, with better ratio precision / computation time.

\section{Appendix B: Circular prolate functions}

Circular prolate functions where invented independently by Slepian (1964) and Heurtley (1964) and have found very interesting applications in Optics, for example for the study of confocal laser modes or wave aberrations. Theses applications of prolate functions in Optics are detailed in the excellent review paper by Frieden (1971). The short presentation of the mathematical properties of circular functions is drawn from Frieden's paper. However, notations have been adapted to fit the present application.

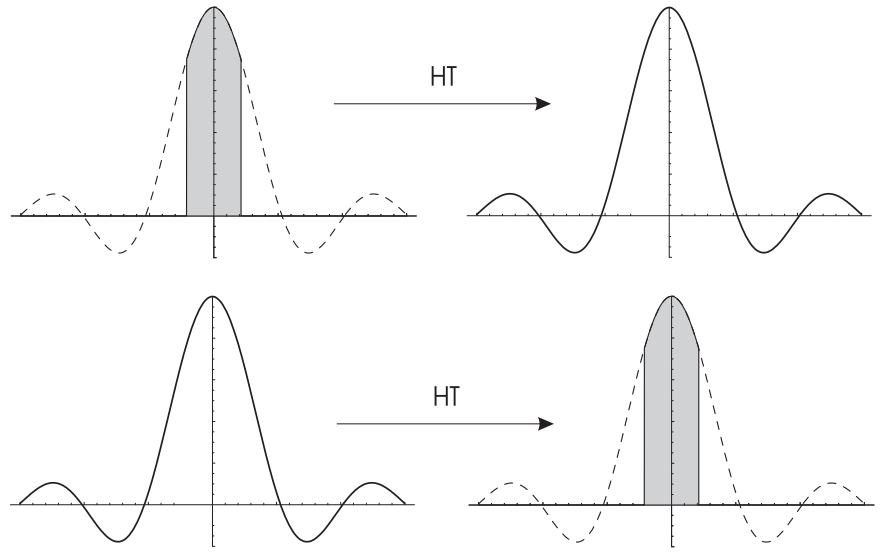

Fig. B.1. Illustration of the properties of prolate function. Top: invariance to the finite Hankel Transform (Eq. (B.1)); the HT of the truncated prolate gives the prolate itself. Bottom: the HT of the entire prolate gives the truncated prolate (Eq. (B.2)).

Circular prolate functions are the circular analogy to the linear prolate function, known since Niven (1880) and rediscovered by Slepian in 1959 (Slepian \& Pollak 1961). Their properties are strictly analogous to those of the linear functions. In the circular case, Hankel Transforms (HT) replace the linear Fourier Transforms (FT). Circular prolate function are defined for any order $N$ of HT, but in the present application we will only need to consider the HT of zero order, involving the Bessel $J_{0}$ function that appears in Fourier Optics for Hankel Transforms. We will omit the subscripts $N$ and $n$ in the notations for clarity (the prolate function for $n=0$ is the only one with maximum at origin).

Circular prolate functions are defined by their invariance to a finite Hankel Transform (the finite HT of a function is the HT of the truncated function):

$$
\int_{0}^{\frac{D}{2}} \Theta(\xi) J_{0}(2 \pi r \xi) 2 \pi \xi \mathrm{d} \xi=\frac{D}{a} \Lambda^{\frac{1}{2}} \Theta\left(\frac{r D}{a}\right) .
$$

The eigenvalue $\Lambda$ and the prolate function $\Theta(r)$ depend on the so-called prolateness parameter $c=\frac{\pi D a}{2}$, that we omit in the notations, for clarity.

Reciprocally, the circular have the following property for the infinite HT:

$$
\begin{aligned}
\int_{0}^{\infty} \Theta(\xi) J_{0}(2 \pi r \xi) 2 \pi \xi \mathrm{d} \xi & =\frac{D}{a} \Lambda^{-\frac{1}{2}} \Theta\left(\frac{r D}{a}\right) \\
& (0 \leq r \leq a / 2) \\
& =0(r>a / 2) .
\end{aligned}
$$

These two properties are the most interesting properties for coronagraphy: the HT of a truncated central part of the prolate function gives the prolate function itself (to normalisation factors). The HT of the entire prolate function gives the same central truncated part. A heuristic illustration of these two properties (Eqs. (B.1) and (B.2)) is given in Fig. B.1.

Performing a finite HT on both side of Eq. (B.1), we have:

$(2 \pi)^{2} \int_{0}^{\frac{D}{2}} \xi \Theta(\xi) K_{0}(\xi, r) \mathrm{d} \xi=\Lambda \Theta(r)$ 
$K_{0}(\xi, r)=\int_{0}^{\frac{a}{2}} \rho J_{0}(2 \pi \rho \xi) J_{0}(2 \pi \rho r) \mathrm{d} \rho$.

The circular prolate function appear to be the eigenfunctions of this integral equation, with eigenvalue $\Lambda$ (we give the kernel expression in Eqs. (A.8) and (A.9)).

A given eigenvalue correspond to a unique mask size $a$ and a unique prolate function, as illustrated in Fig. B.2 (usually this curve is drawn as a function of the prolateness parameter $c=$ $\frac{\pi D a}{2}$, but for clarity we consider it as a function of the mask size (the telescope diameter $D$ is a constant).

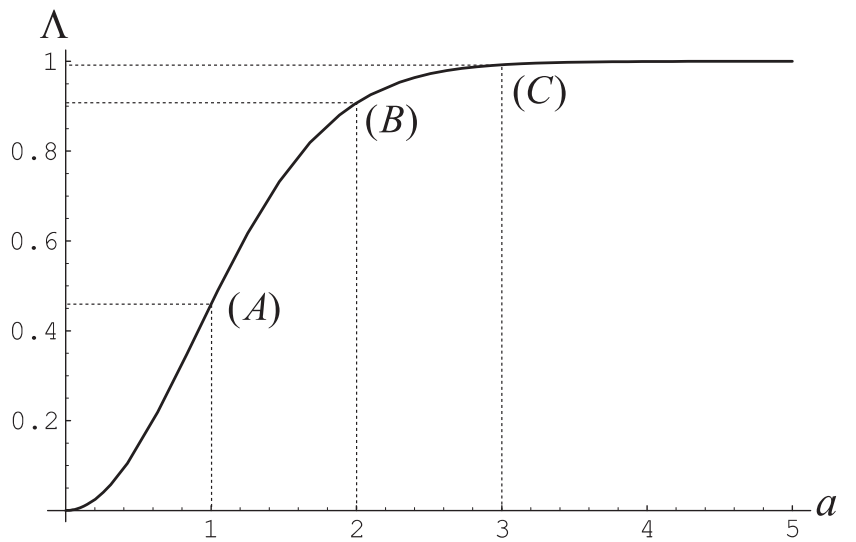

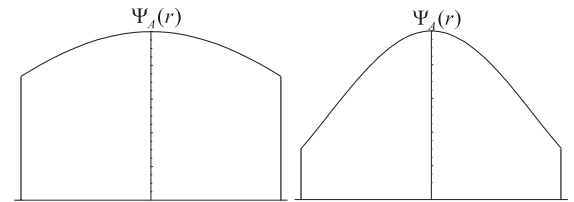

(A)

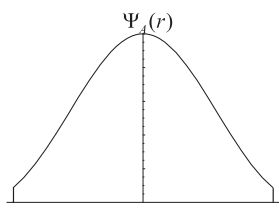

$(C)$
Fig. B.2. Eigenvalue $\Lambda$ as a function of the mask size $a$, and corresponding prolate functions of zero order $\Theta(r) / \Theta(0)$ (normalized). The closest to 1 the eigenvalue, the stronger the apodization. Three examples are given for three eigenvalues. The corresponding apodization functions are given in amplitude.

The double orthogonality property of the linear prolates also exists for the circular prolates of orders $m$ and $n$ :

$\int_{0}^{\frac{D}{2}} r \Theta_{n}(r) \Theta_{m}(r) \mathrm{d} r=\Lambda_{n} \delta_{m n}$

$\int_{0}^{\infty} r \Theta_{n}(r) \Theta_{m}(r) \mathrm{d} r=\delta_{m n}$

where $\delta_{m n}$ is the Kronecker delta.

This property is useful for the computation of the transmissions of the prolate apodizations (with $m=n=0$ ).

An other fundamental property is the fractional energy within $0 \leq r \leq r_{0}$ which finds a simple expression:

$e=\frac{\int_{0}^{\frac{a}{2}} r\left|\Theta_{N, n}(r)\right|^{2} \mathrm{~d} r}{\int_{0}^{\infty} r\left|\Theta_{N, n}(r)\right|^{2} \mathrm{~d} r}=\Lambda_{N, n}$.

Moreover, the maximum fractional energy correspond to $N=0$ and $n=0$ (the case considered in the present study), and we have (Frieden 1971):

$e_{\max }=\Lambda$.
This is why we consider only for apodization the functions corresponding to $N=0$ and $n=0$. $\left(\Theta_{0}(r)\right.$ is also the only one with its maximum at the origin).

\section{Appendix C: Computation of the circular prolate functions}

The circular prolate functions used in this paper can be defined in terms of Slepian's "generalized prolate spheroidal functions" $\varphi_{N, n}(c, x)($ Slepian 1964):

$\Theta_{N, n}(c, r)=\sqrt{\frac{2 \Lambda_{N, n}(c)}{D}} \frac{\varphi_{N, n}\left(c, \frac{2 r}{D}\right)}{\sqrt{r}}$.

Here $N$ is the order of the Hankel transform, $n$ the order of the prolate function, and $c=\pi D a / 2$ the prolateness parameter. In this paper we are primarily interested in the case $N=n=0$, and for convenience have generally omitted these labels.

The functions $\varphi_{N, n}(c, x)$ are solutions of a generalized spheroidal differential equation,

$\left(1-x^{2}\right) \varphi^{\prime \prime}(x)-2 x \varphi^{\prime}(x)+\left(\chi-c^{2} x^{2}+\frac{\frac{1}{4}-N^{2}}{x^{2}}\right) \varphi(x)=0$

with eigenvalues $\chi=\chi_{N, n}(c)$. They are conventionally normalized so that

$\int_{0}^{1} \varphi_{N, n}(c, t)^{2} \mathrm{~d} t=1$.

When $c=0$, Eq. (C.2) is satisfied by the functions $T_{N, n}(x)$, defined by

$T_{N, n}(x)=x^{N+\frac{1}{2}}{ }_{2} F_{1}\left(-n, n+N+1 ; N+1 ; x^{2}\right)$,

where ${ }_{2} F_{1}(a, b ; c ; z)$ is Gauss' hypergeometric function (Abramowitz \& Stegun 1965), and the eigenvalue is $\chi_{N, n}(0)=$ $(N+2 n+1 / 2)(N+2 n+3 / 2)$. The $T_{N, n}(x)$ satisfy the three-term recurrence relation

$x^{2} T_{N, n}=\gamma_{N, n}^{1} T_{N, n+1}+\gamma_{N, n}^{0} T_{N, n}+\gamma_{N, n}^{-1} T_{N, n-1}$,

where

$\gamma_{N, n}^{1}=-\frac{(n+N+1)^{2}}{(2 n+N+1)(2 n+N+2)}$,

$\gamma_{N, n}^{0}=\frac{1}{2}\left(1+\frac{N^{2}}{(2 n+N)(2 n+N+2)}\right)$,

$\gamma_{N, n}^{-1}=-\frac{n^{2}}{(2 n+N)(2 n+N+1)}$.

For general $c$, the functions $\varphi_{N, n}(c, x)$ are expanded in a series of the $T_{N, k}(x)$ :

$\varphi_{N, n}(c, x)=\sum_{k=0}^{\infty} d_{k}^{N, n}(c) T_{N, k}(x)$.

Substituting this expansion into Eq. (C.2) and using Eq. (C.5), we obtain a three-term recurrence for the series coefficients $d_{k}^{N, n}(c)$ :

$$
\begin{array}{r}
c^{2} \gamma_{N, k-1}^{1} d_{k-1}^{N, n}+\left(c^{2} \gamma_{N, k}^{0}+\chi_{N, k}(0)-\chi_{N, n}(c)\right) d_{k}^{N, n} \\
+c^{2} \gamma_{N, k+1}^{-1} d_{k+1}^{N, n}=0
\end{array}
$$


The eigenvalues $\chi_{N, n}(c)$ and the coefficients $d_{k}^{N, n}(c)$ can be computed from this equation using the standard tridiagonal matrix or continued fraction methods (Falloon 2001). For $x>1$, $\varphi_{N, n}(c, x)$ can be computed using the series

$\varphi_{N, n}(c, x)=\frac{1}{\gamma_{N, n}(c)} \sum_{k=0}^{\infty} d_{k}^{N, n}(c) \frac{J_{2 k+N+1}(c x)}{\left(\begin{array}{c}k+N \\ k\end{array}\right) \sqrt{c x}}$

where

$\gamma_{N, n}(c)=\frac{c^{N+\frac{1}{2}} d_{0}^{N, n}}{2^{N+1} \Gamma(N+2)}\left(\sum_{k=0}^{\infty} d_{k}^{N, n}(c)\right)^{-1}$.

The eigenvalues of the integral equation (Eq. (B.3)), $\Lambda_{N, n}(c)$, can be found using

$\Lambda_{N, n}(c)=c \gamma_{N, n}(c)^{2}$.

\section{References}

Abe, L., Vakili, F., \& Boccaletti, A. 2001, A\&A, 374, 1161

Abe, L., Domiciano de Souza, J., Vakili, F., \& Gay, J. 2003, A\&A, in press

Abramowitz, \& Stegun 1965, Handbook of mathematical functions (ed. Dover, New-York)

Aime, C., Soummer, R., \& Ferrari, A. 2001, A\&A, 379, 697

Aime, C., Soummer, R., \& Ferrari, A. 2002, A\&A, 389, 334

Baudoz, P., Rabbia, Y., \& Gay, J. 2000a, A\&AS, 141, 319

Baudoz, P., Rabbia, Y., Gay, J., et al. 2000b, A\&AS, 145, 341

Beuzit, J.-L., Mouillet, D., Lagrange, A.-M., \& Paufique, J. 1997, A\&AS, 125,175

Boccaletti, A., Moutou, C., Labeyrie, A., Kohler, D., \& Vakili, F. 1998, A\&AS, 133, 395
Bonneau, D., Josse, M., \& Labeyrie, A. 1975, in ASSL 54, Image Proc. Techniques in Astronomy, 403

Falloon, P. 2001, Master's Thesis, University of Western Australia Frieden, B. R. 1971, Progress in Optics, ed. E. Wolf, vol. 9

Gay, J., \& Rabbia, Y. 1996, C. R. Acad. Sci. Paris, 322, Ser. II b, 265

Goodman, J. 1996, Introduction to Fourier Optics, ed. M. G. Hill

Guyon, O., \& Roddier, F. 2000, in Proc. of SPIE, 40006

Heurtley, J. 1964, Proc. Symp. on Quasi Optics, New York, 367

Malbet, F. 1996, A\&AS, 115, 161

Mayor, M., \& Queloz, D. 1995, Nature, 378, 355

Mouillet, D. 1997, Ph.D. Thesis, Laboratoire d'Astrophysique de l'Observatoire de Grenoble, France

Mouillet, D., Lagrange, A.-M., Beuzit, J.-L., \& Renaud, N. 1997, A\&A, 324, 1083

Nisenson, P., \& Papaliolios, C. 2001, ApJ, 548, L201

Niven, C. 1880, Phil. Trans. Roy. Soc. London, Ser. A, 171, 117

Roddier, C., Roddier, F., Martin, F., Baranne, A., \& Brun, R. 1980, J. Opt., 11, 149

Roddier, F., \& Roddier, C. 1997, PASP, 109, 815

Rouan, D., Riaud, P., Boccaletti, A., Clénet, Y., \& Labeyrie, A. 2000, PASP, 112, 1479

Slepian, D. 1964, Bell Syst. Tech. J., 43, 3009

Slepian, D., \& Pollak, H. O. 1961, Bell Syst. Tech. J., 40, 43

Smith, B. A., \& Terrile, R. J. 1984, Science, 226, 1421

Soummer, R., Aime, C., \& Falloon, P. 2002a, in Astronomy With High Contrast Imaging: from planetary systems to avtive galactic nuclei, ed. C. Aime, \& R. Soummer (EAS Publication Series)

Soummer, R., Aime, C., Ferrari, A., \& Falloon, P. 2002b, in Proc. SPIE Vol. 4860, High Contrast Imaging for Exoplanet Detection, ed. A. B. Schultz, \& R. G. Lyon, vol. 4860

Watson, S. M., Mills, J. P., Gaiser, S. L., \& Diner, D. J. 1991, Appl.Opt., 30, 3253

Wolfram, S. 1999, The Mathematica book, Fourth Edition (Cambridge University Press ed.)

Wynne, C. G. 1979, Opt.Commun., 28, 21 